\title{
Constructing the Knowledge Model in ERP Implementation
}

\author{
Jiangao Deng and Yijie Bian \\ Business School, Hohai University, Nanjing 210098, Jiangsu, P.R.China \\ djgwmdkx@163.com byj@hhu.edu.cn
}

\begin{abstract}
Successful knowledge transfer is an effective guarantee for ERP implementation. This paper introduces knowledge types used in ERP implementation, sorts out structure components of such knowledge, analyses the key points of knowledge and its transfer involved in the three ERP implementation entities, and then constructs three-dimensional knowledge models at two main ERP implementation stages, confirming stage and implementing stage, at last it gives a detailed decomposition.
\end{abstract}

Keywords: ERP, Knowledge transfer, Knowledge model

\section{INTRODUCTION}

As an important means to facilitate and realize innovation in system, technology and management, informationization has become a popular strategy among enterprises. ERP (Enterprise Resource Planning), which reflects today's most advanced enterprise management theory, has realized an optimum management of enterprise's resources, by offering the best scheme for informationization integration [1]. Nevertheless, whether an enterprise can integrate information technology with its organization, overall management and culture, in order to advance its scientific management and its core competition ability, lies on the effective knowledge management during the ERP implementation process. As we all know, most enterprises, who invested in informationization, usually fail to fully realize their original purpose. Therefore, a research on knowledge models in ERP implementation becomes necessary. According to several years of experience in ERP implementation, the author of this paper sorts out knowledge types and puts forth a 3-dimensional knowledge model (3-D KM) in ERP implementation, in hopes of benefiting the ERP implementation practice.

\section{ERP IMPLEMENTATION KNOWLEDGE TYPES}

ERP Implementation Knowledge (ERP-IK), which is distributed among all ERP implementation participating parties, falls into two categories, namely explicit knowledge and tacit knowledge. 
Explicit knowledge refers to the reports and the lectures from experts, manuals on software and hardware, and other documents. Explicit knowledge is quite general, to which enterprises can have easy access at a certain cost.

Tacit knowledge, on the contrary, is quite empirical, which is hard to be materialized or transmitted in words or formulas, as well as in conventional ways. Tacit knowledge is mainly embodied in 3 aspects: Firstly, the methodology, templates and technical knack used in the ERP implementation process; Secondly, management ideas, business process designs and professional experience incarnated in an ERP software; and thirdly, the impact of ERP implementation exerted on an enterprise organization, management system and culture[2].

\section{STRUCTURE COMPONENTS OF ERP-IK}

In the ERP implementation process, knowledge needing transfer is in various forms and at different levels of difficulty, according to which, structure components of ERP-IK can be divided into two parts: Structure components of knowledge type and Structure components of knowledge state [3-4].

Table 1. Structure Components of ERP-IK

\begin{tabular}{l|l|l|l}
\hline & & Name & Symbol \\
\hline \multirow{4}{*}{$\begin{array}{l}\text { Structure } \\
\text { Components }\end{array}$} & \multirow{4}{*}{$\begin{array}{l}\text { Structure Components } \\
\text { of Knowledge Type }\end{array}$} & Data Knowledge & DK \\
\cline { 3 - 4 } & & Program Knowledge & PK \\
\cline { 3 - 4 } & & Function Knowledge & FK \\
\cline { 3 - 4 } & & Management Knowledge & MK \\
\cline { 3 - 4 } & \multirow{3}{*}{$\begin{array}{l}\text { Structure Components } \\
\text { of Knowledge State }\end{array}$} & Formalized Knowledge & FOK \\
\cline { 3 - 4 } & & Renewed Knowledge & RK \\
\cline { 3 - 4 } & & Emerging Knowledge & EMK \\
\cline { 3 - 4 } & & Experiential Knowledge & EXK \\
\hline
\end{tabular}

DK is the most fundamental data in ERP implementation. PK refers to the rules, regulations, and protocols. FK is the efficient workflow and process. MK is the logical and systematic Management models and knowledge systems. IK is the effective application of administration knowledge. RK means renewed knowledge. FOK is the formalized knowledge, which belongs to explicit knowledge. EMK is the emerging knowledge, which is composed of both explicit and tacit knowledge. EXK is the experiential knowledge, which belongs to tacit knowledge.

\section{KNOWLEDGE TRANSFER AMONG ERP IMPLEMENTATION ENTITIES}

There are the three organizational entities in ERP implementation, including ERP Applying Enterprise (EAE), ERP Software Provider (ESP) and Professional 
Consultant (PC). The most important work of ERP implementation is to transfer technology and knowledge among these three entities [5].

Knowledge transferred from EAE to ESP and PC includes knowledge of the profession, of enterprise demands and individualized demands, etc. Knowledge transferred from ESP to EAE and PC includes knowledge of ERP theories, of project solutions, of project implementation methodology, etc. Knowledge transferred from PC to EAE and ESP includes knowledge of judgment of enterprise demands, of venture evaluation, etc.

The knowledge mentioned above is disseminated in its various structure components, all of which transfer at a different degree of difficulty. As a result, it is necessary to construct an ERP implementation knowledge model (ERP-IKM).

\section{CONSTRUCTING THE ERP IMPLEMENTATION KNOWLEDGE MODEL}

By summarizing CIM enterprise modeling theories, especially the CIM-OSA modeling theory and Professor Congdong Li's modeling thought [6-9]. The paper puts forward a three-dimensional ERP-IK model (3-D ERP-IKM). This 3-D ERPIKM, which describes ERP-IK belonging to the three entities, forms the basic theory of ERP-IK transfer. The main panel of the model is composed of knowledge type components and knowledge state components, which describe the ERP-IK contents, forms, transfer difficulty, and transfer strategy at different implementation stages.

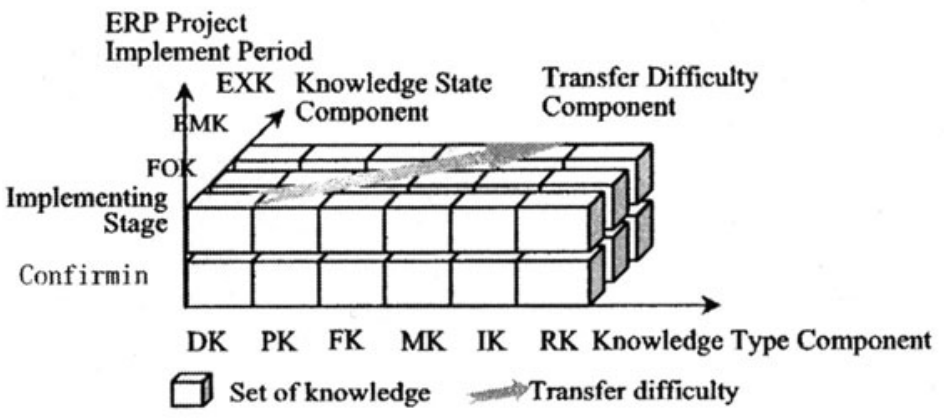

Figure 1. 3-D ERP-IKM

The knowledge transfer difficulty component is also one kind of ERP-IK components. It is intrinsic feature of knowledge and its causal ambiguity that causes great obstacles to knowledge transfer and duplication.

Now, the knowledge transfer difficulty component in this 3-D ERP-IKM rises to such occasions by describing the characteristics of such implicit knowledge explicitly. In the 3-D ERP-IKM, the X-axis shows the six structure components of knowledge type, namely DK, PK, FK, MK, IK and PK, with an increasing transfer difficulty; the 
Y-axis shows the three structure components of knowledge state, namely FOK, EMK and EXK, with an increasing transfer difficulty.

\subsection{Further Development of ERP-IKM}

In order to ensure a successful knowledge transfer in ERP implementation, this paragraph puts forward ERP-IKM at the confirming stage and implementing stage of the project respectively [10-11].

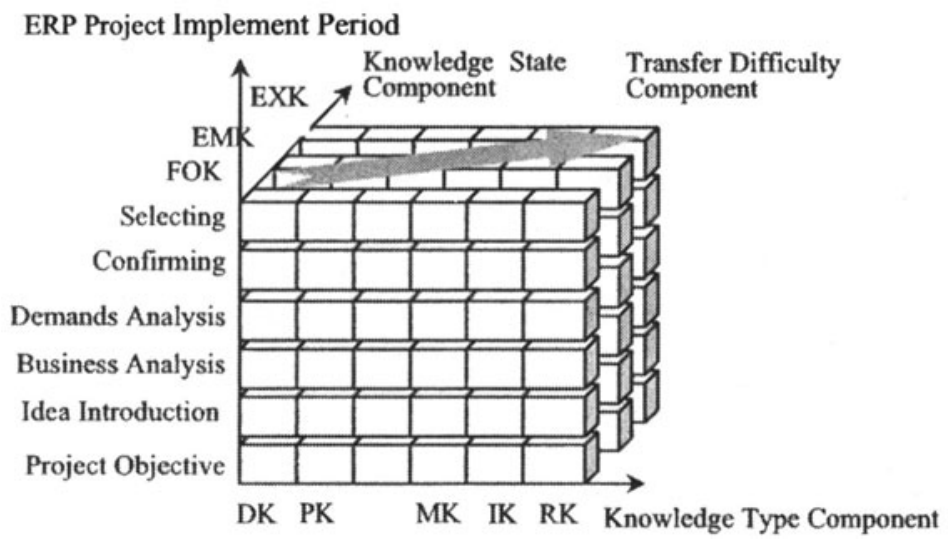

Figure 2. ERP-IKM at the Confirming Stage Knowledge

Figure 2 describes the main contents of ERP-IK at the confirming stage of the project, including knowledge of the project's objectives (enterprise background, project's objective), of ERP ideas (basic conceptions about ERP, preliminary functions of ERP modules), of enterprise transactions (procedures, functions, and existing problems of enterprise transactions), of the project's demands, of ERP software providers, and knowledge of project's management, etc.

Figure3 describes the main contents of ERP-IKM at the implementation stage of the project, including knowledge of implementation preparation, of business blueprint, and of specific implementation, etc. 


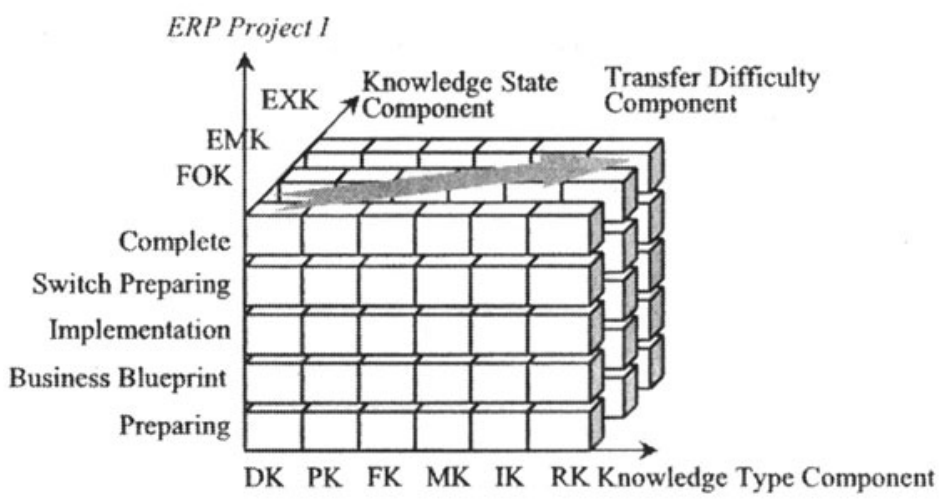

Figure 3. ERP-IKM at the Implementing Stage

\section{CONCLUSIONS}

To sum up, the course of ERP implementation is the process of knowledge transfer. In such a course, several entities are involved, as well as the two knowledge types, i.e. explicit knowledge and tacit knowledge. Having analyzed the structure components of ERP-IK, and carried out a preliminary research on them, this paper puts forward a three-dimensional knowledge management model, which is used in turn to instruct ERP implementation practice. However, there are still some important aspects which have not been explored, for example, knowledge transfer system involved with software providers, professional consultants and implement enterprises, which can also contribute to a successful ERP implementation.

\section{REFERENCES}

1. W. Skokl and M. Legge, Evaluating enterprise resource planning systems using an interpretive approach, Knowledge and Process Management. Volume 9, Number 2, pp.72-82, (2003).

2. L. Jeffrey, F. Cummings, and B. Teng, Transferring R\&D knowledge: the key factors affecting knowledge transfer success, Journal of Engineering and Technology. Volume 20, Number. 2, pp.39-68, (2003).

3. K. Joshi, S. Sarker, and S. Sarker, Knowledge transfer within information systems development teams: Examining the role of knowledge source attributes, Decision Support Systems. Volume 41, Number 2, pp.456-460, (2006). 
4. M. Alavi and D. Leidner, Review knowledge management and knowledge management systems conceptual foundations and research issues, MIS Quarterly. Volume 4, Number 25, pp.11-20, (2001).

5. C. Mary, D. Jones, M. Cline, and H. Ryan, Exploring knowledge sharing in ERP implementation: an organizational culture framework, Decision Support Systems. Volume 41, Number 2, pp.411-434, (2006).

6. A. Vincent, D. Mabert, A. Soni, and M. Venkataramanan, Model based interpretation of survey data: a case study of enterprise resource planning implementations, Mathematical and Computer Modeling. Volume 44, Number 1, pp.16-29, (2006).

7. H. Du, C. Li, and X. Li, Knowledge transfer architecture for ERP implementation, Journal of Industrial Engineering. Volume 19, Number 2, pp.110-113, (2005).

8. S. Newell, J. Huang, R. Galliers, and S. Pan, Implementing enterprise resource planning and knowledge management systems in tandem: fostering efficiency and innovation complementarity, Information and Organization. Volume 9, Number 23, pp.32-46, (2003).

9. E. Daniel and O. Leary, Knowledge management across the enterprise resource planning systems life cycle, International Journal of Accounting Information System. Volume 8, Number 5, pp.99-100, (2002).

10. F. Eppler, J. Martin, and O. Sukowski, Managing team knowledge: Core process, tools and enabling factors. European Management Journal. Volume 10, Number 18, pp.334$341,(2000)$.

11. D. Malone, Knowledge management: a model for organizational learning, International Journal of Accounting Information Systems. Volume 3, Number 2, pp.111-123, (2002). 